\section{¿Por qué AICLE? Un análisis de la literatura desde la perspectiva de los docentes de materias no lingüísticas}

\author{
Maria Elisabetta Porcedda (D), Juan González-Martínez \\ Rebut: 27/08/2019 Acceptat: 15/10/2019
}

\title{
Resumen
}

Desde su nacimiento en 1994 hasta ahora, el modelo AICLE (Aprendizaje Integrado de Contenidos y Lenguas Extranjeras) ha sido conceptualizado e implementando de diferentes modos, según las políticas de los diferentes gobiernos, o de las escuela, o de las propias actitudes de los docentes interesados, lo que ha causado un margen de vaguedad en su definición. Esto es muy importante, ya que la adopción de CLIL por parte de la Unión Europea para apoyar el objetivo del multilingüismo se ha centrado en los resultados lingüísticos en varios niveles y, por lo tanto, en las investigaciones y encuestas llevadas a cabo; sin embargo, las pràcticas CLIL las implementan principalmente docentes de asignaturas no lingüísticas, que necesitan ver la efectividad de su perspectiva, no solo desde el lingüístico. Este documento revisa la literatura y encuentra la definición de CLIL como "entorno abierto de educación", definitivamente adecuado para los profesores de cualquier asignatura no lingüística para poder participar en su implementación. También se subraya, teniendo en cuenta las directivas europeas, cómo el CLIL, en particular si se enseña a través de herramientas ofrecidas por las nuevas tecnologías, tiende a facilitar el aprendizaje global de los estudiantes y su ciudadanía europea, así como a ser el primer paso para uno nuevo modelo de práctica escolar.

Palabras Clave: implementación AICLE, rol docente, evaluación, entorno de aprendizaje, ciudadanía europea.

\footnotetext{
Abstract

Since its birth in 1994 until now, CLIL (Content and Language Integrated Learning) has been seeing and implementing in different ways, depending on government or school policies or stakeholders' attitudes, which has caused a margin of vagueness about its definition. This is very important, because the European Union adoption of CLIL to support the aim of multilingualism has focused on the linguistic results at several levels and so the researches and surveys, although it is implemented mainly by subject teachers, who need to see the effectiveness from their point of view, not only linguistic. This paper reviews the literature, finding out the definition of CLIL as 'open environment of education', definitely suitable for subject teachers in order to be involved in its implementation. It is also underlined, taking into account the European Directives, how CLIL, in particular if taught through tools offered by new technologies, tend to achieve the students' global growth and their European citizenship, as well as to be the first step for a new kind of schooling.
} 
Keywords: CLIL implementation, teacher role, student evaluation, classroom environment, European citizenship.

\section{Introducción}

Según la Comisión Europea, en casi toda Europa el Aprendizaje Integrado de Contenidos y Lenguas Extranjeras (AICLE; también conocimo como CLIL, por las siglas inglesas Content and Language Integrated Learning) se considera una opción interesante para revitalizar tanto el aprendizaje como la enseñanza (Marsh, 2002). Opción que puede contribuir a modernizar los sistemas escolares a través de un nuevo enfoque (R . Kiely, 2011; Banegas, 2012), que también incluye usos tecnológicos (Meyer, 2010); pero, sobre todo, una opción que incide directamente la competencia clave del aprendizaje de lenguas extranjeras (Resolution, 2008). Bajo este enfoque, una o más asignaturas no lingüísticas se imparten en una lengua extranjera (o minoritaria), principalmente inglés, en diversas proporciones en función de las políticas estatales o de las consideraciones de cada escuela, con el fin de lograr el dominio de L2 al final de la escuela secundaria (Eurydice, 2017a). Y, como consecuencia de lo anterior, se espera que los profesores de materias no lingüísticas alcancen un nivel adecuado de competencia en la L2 (C1 o B2 del MCER) y que dominen las estrategias del AICLE, sobre todo en su formación universitària (inicial o continua), para poder lograr resultados tanto en los contenidos impartidos y en la L2 utilizados para la enseñanza, solos o en equipos con profesores de idiomas (Banegas, 2012; Eurydice, 2017a).

Esta formación metodológica y lingüística, como las mismas investigaciones sobre la definición del AICLE, o las diferentes formas en que AICLE se ha experimentado en diferentes países y su eficàcia, se ha centrado demasiado a menudo en la adquisición, temas y objetivos de L2, y por ello se ha abordado de modo general desde la perspectiva de la lingüística aplicada, la cual no deja de ser una visión segmentada. Por ello, este artículo ofrece, a partir de una revisión de la literatura, una panorámica sobre las investigaciones en AICLE, con el fin de conocer los puntos fuertes y las oportunidades de este enfoque que los docentes de materias no lingüísticas pueden considerar adecuados, en especial por lo que respecta a los procesos de aprendizaje ligados a los contenidos disciplinares.

\section{Preguntas sobre la investigación}

Desde su adopción en Europa en los años 90, AICLE, debido a su soporte válido para el objetivo del multilingüismo, ha sido muy bien acogido por los profesores e investigadores de lenguas extranjeras; y son estos quienes han hecho implícita su importancia también para los docentes de materias no lingüísticas, que deben poner aplicarlo con una enorme carga de trabajo más. Por ello, con el fin de señalar la importancia de AICLE para el profesorado en general (y no solo para los docentes de lenguas), es relevante responder a las siguientes preguntas:

- ¿Existe una definición de AICLE que pueda ser atractiva para el profesorado en general, con un doble papel, y que apunte a la evaluación de los aprendizajes de los estudiantes tanto en relación con el contenido como con la lengua extranjera?

- ¿Cómo se ha venido aplicando el AICLE, en particular en Europa, hasta ahora? 
- ¿Hay resultados de la enseñanza del AICLE que puedan ser considerados relevantes y tentadores para los docentes de materias no lingüísticas?

\section{Metodología}

Esta revisión sistemática de la literatura ha sido llevada a cabo desde el punto de vista de los profesores europeos de materias no lingüísticas, con el fin de señalar el significado y la importancia de implementar el AICLE, como resultado del análisis de los propios fundamentos teóricos, y mediante la revisión de la documentación esencial y accesible en un procedimiento sistemático, que incluye una estrategia de búsqueda y un análisis categorial planeado, ligado a nuestras preguntas de investigación (Okoli y Schabram, 2010).

Partimos de la consideración de que el AICLE ha sido ampliamente investigado y, por tanto, disponemos de una gran cantidad de documentos y resultados; por ello, los datos tomados en cuenta aquí derivan en primer lugar de la busqueda y el análisis de los documentos de la Comisión Europea y del Consejo Europeo, que ofrecen referencias comunes acerca de las recomendaciones de objetivos a alcanzar en los estudios y del proceso en curso de la práctica del AICLE en todos los Estados. En segundo lugar, una selección de artículos revisados por investigadores relevantes o artículos experimentales relevantes y obtenidos a través de tres repositorios académicos (Scopus, Educational Resources Information Center (ERIC) y Google Scholar). Finalmente, las definiciones y prácticas AICLE provienen de los documentos fundacionales de David Marsh, Do Coyle o Phil Ball; o, por las investigaciones sobre AICLE de Christiane Dalton-Puffer y Ana Llinares.

\section{4. ¿Qué es AICLE?}

En 1994, AICLE nació de la colaboración entre la Universidad finlandesa de Jyväskylä y la Plataforma Europea para la educación holandesa, de la mano de David Marsh y Anne Maljers. Esta es su definición: "AICLE se refiere a situaciones donde las asignaturas, o parte de las asignaturas, se enseñan a través de una lengua extranjera con un objetivo doble, especificamente el aprendizaje de contenidos y el aprendizaje simultáneo de una lengua extranjera" (recogido en Biçaku, 2011, p. 3822). Como se ve, es originalmente un 'paraguas' abierto (Dalton-Puffer, Llinares, Lorenzo \& Nikula, 2014; Eurydice, 2017b), bajo el cual pueden encontrarse contenidos diversos y cualquier idioma, minoritario o hablado en Europa (Eurydice, 2017a).

AICLE se ha extendido en casi toda Europa, gracias a los objetivos educativos de la Comisión Europea (1995) y en particular para generalizar inicialmente el dominio de tres lenguas comunitarias, aunque luego de dos (Barcelona European Council, 2002); pero también gracias a la existencia de países multilingües (Eurydice, 2017a), como España (Guillamón-Suesta, Luisa \& Renau, 2015), y/o contextos escolares que apuestan decisivamente por la L2 (Eurydice, 2017a; Bruton, 2011), a menudo adoptando el 'AICLE fuerte', muy cerca de la Canadian Content-Based Instruction (Pérez Cañado, 2016; Muñoz-Luna, 2014; Banegas, 2012; Dalton-Puffer, 2011).

Puede parecer que la preocupación era encontrar una estrategia para enseñar idiomas el mayor tiempo posible en la escuela, para alcanzar las metas de este objetivo europeo; de hecho, la mayoría de los estudios e investigaciones hasta el momento se centran en la adquisición de L2 como 'enfoques centrados en la lingüística', donde el 'contenido puede ser visto como una herramienta de mediación para el aprendizaje de idiomas' (Banegas, 2012, p.118), si bien hay pocas publicaciones no en Inglés sobre el aprendizaje de contenido en el AICLE (Dalton-Puffer, Llinares, Lorenzo \& Nikula, 2014). 
Sin embargo, el contenido al principio fue subrayado en el marco de las 4C (Coyle, 1999), un cronograma de proyectos de objetivos en las áreas de contenido, comunicación, cognición y cultura para las clases de AICLE, donde el lenguaje es 'una herramienta' para el éxito del aprendizaje del alumnado (Coyle, 2002; Banegas, 2012). Desde entonces, AICLE ha comenzado a convertirse en una estrategia para aumentar las capacidades comunicativas, cognitivas e interculturales de los estudiantes, enseñadas de manera diferente (Coyle, 2002; Meyer, 2010) y adoptando principalmente un enfoque socio-constructivista (Muñoz-Luna, 2014; Meyer, 2010), en particular para aprovechar las 'oportunidades de interacción entre los participantes' a través de las teorías basadas en input y scaffolding (Nikula, Dalton-Puffer \& García, 2013), a menudo enriquecidas por el uso de TIC (Marsh \& Frigols Martín, 2012; Ramírez-Verdugo, 2012; Cinganotto, 2016).

Con todo, el AICLE siempre ha recibido la atención amplia en términos de investigación sobre sus funciones de, para y a través del aprendizaje (Coyle, 2002), desde la lingüística aplicada (por ejemplo, Dalton-Puffer, 2007; Llinares \& Whittaker, 2007; Llinares \& Morton, 2010; Muñoz-Luna, 2014) para apoyar a los maestros en su práctica. Para este tipo de estudios, también, la definición del AICLE parece difícil y la conclusión podría ser un enigma (Dalton-Puffer et al, 2014; Pérez Cañado, 2016), debido a la enorme necesidad de nuevas investigaciones, involucrando sin duda a profesores (Nikula et al, 2013.); o realmente un paraguas (Dalton-Puffer, Llinares, Lorenzo \& Nikula, 2014) o un 'ambiente de aprendizaje poderoso' (Marsh \& Frigols Martín, 2012, p.7), en virtud del cual podemos encontrar diferentes estrategias, diferentes tipos de contenido y idiomas insertados y en diferentes porcentajes.

\section{Aplicación del AICLE}

Con el fin de analizar los datos sobre la ejecución europea del AICLE, es útil saber qué idioma adicional se nos enseña a través de este. La política de la Unión Europea nunca ha sugerido la enseñanza de un idioma en particular entre los mayoritarios, y concede la libertad de utilizar el AICLE también para las minorías (Eurydice, 2017a); per el inglés es, con mucho, el idioma más estudiado en casi todos los países europeos (Eurydice, 2017a; Marsh \& Frigols Martín, 2012), hasta el punto de que a menudo es más probable hablar de CEIL (Content and English Integrated Learning) en lugar de AICLE o CLIL (DaltonPuffer, 2011). Por tanto, a pesar de las intenciones por parte de la Unión Europea sobre la propagación del multilingüismo (Council Resolution 2008), el afianzamiento del inglés como lingua franca europea podría estarse impulsando también a través de la puesta en práctica del AICLE (Dalton-Puffer, 2011; Marsh \& Frigols Martín, 2012). Sin embargo, el francés y alemán, pero también el español son ampliamente objetivos dentro de la disposición del AICLE (Eurydice, 2017a), ya que las lenguas minoritarias son mayormente enseñadas en los países donde existe más de un idioma oficial (Eurydice, 2017a), como en España (Eurydice, 2017a).

Aquí vale la pena subrayar que la función del AICLE es no ser practicado en su totalidad en el idioma de destino como en la IBC (Instrucción Basada en Contenidos), aunque suced así; sino en un porcentaje de las horas curriculares de materias no lingüísticas, por lo general menos del $50 \%$ por asignatura y año (Nikula et al., 2013). Por tanto, si la lengua AICLE es la propia lengua extranjera curricular, los estudiantes se colocan bajo una exposición más amplia de este a diferencia de los estudiantes sin AICLE (Bruton, 2011; Dalton-Puffer, 2011; Marsh \& Frigols Martín, 2012), según lo recomendado por el Consejo Europeo (Council Resolution 2008).

Otro asunto importante es la edad inicial de los estudiantes AICLE en Europa, ya que la Unión Europea busca 'mejorar el dominio de las competencias básicas, en particular al enseñar al menos dos lenguas extranjeras desde una edad muy temprana' (Barcelona European Council, 2002, p. 19). La situación es compleja, debido a la extrema heterogeneidad de las diferentes implementaciones no solo entre países, sino entre regiones y también entre las políticas escolares, debido a que solo unos pocos países tienen 
una política estatal precisa para AICLE y a que en otros, a pesar de que hay un marco general AICLE, a menudo no son ni recomendaciones centrales ni se da una aplicación generalizada (Eurydice, 2017a). Es lo que Dalton-Puffer (2007) llama una brecha entre las actividades locales fundamentales y el nivel supranacional, es decir, entre las acciones de los maestros o escuelas individuales y las iniciativas transnacionales con el apoyo de organizaciones europeas (Czura \& Papaja, 2013). En cualquier caso, se ha investigado sobre AICLE tanto en primaria (por ejemplo, Pfenninger, 2014; Ramírez-Verdugo, 2012), como secundaria (ver Nikula et al, 2013; Por ejemplo, Czura \& Papaja, 2013; Agolli, 2015; Cinganotto, 2016; Guillamón-Suesta et al, 2015; Llinares \& Whittaker, 2007; Van Kampen, Admiraal \& Berry, 2016; Doiz, Lasagabaster \& Sierra, 2014; Goris, Denessen \& Verhoeven, 2017; Fokides \& Zampouli, 2017; Dallinger, Jonkmann, Hollm \& Fiege, 2016; Hüttner \& Smit, 2017), y en ocasiones a nivel universitario (Gaisch, Pisón, Hrušková, Krátká \& Mádlová, 2017; Del Carmen \& Ribeiro, 2015). Y los resultados revelan que la edad temprana no es factor clave en la adquisición de destrezas lingüísticas (Pfenninger, 2014), ya que, en el aprendizaje de idiomas, generalmente los estudiantes mayores de escuelas secundarias son más eficaces debido a sus mayores capacidades cognitivas (Pfenninger, 2014; Lorenzo, Casal \& Moore, 2010); por ello la propia aplicación del AICLE ayuda a incrementar y hacer apto el contenido del aprendizaje (Meyer, 2010).

Ahora debemos revisar la literatura sobre los contenidos impartidos a través de AICLE. Como se sabe, cualquier materia no lingüística o planteamiento interdiciplinar (ver Banegas, 2012; Coyle, 2015) es bienvenido en una propuesta AICLE (Eurydice, 2017a); sin embargo, es obvio que el proceso de aprendizaje a través de un idioma distinto al habitual será también particular (Marsh \& Frigols Martín, 2012). De hecho, AICLE sería una manera de mejorar las habilidades de los estudiantes no solo en lenguas extranjeras (Marsh \& Frigols Martín, 2012), sino también en el lenguaje académico disciplinar (Marsh, 2002; Dalton-Puffer, 2007; Kiely, 2011; Meyer, Coyle, Halbach, Schuck \& Ting, 2015); así como también aumentaría la profundización de los temas de una manera multilingüe y multicultural (Marsh, 2002; Marsh \& Frigols Martín, 2012; Coyle, 2015; Czura \& Papaja, 2013), como recomienda el Consejo de Europa (por ejemplo: Barcelona European Council, 2002; Council Resolution 2008; Council Recommendation 2012). Con ello, además, los estudiantes transitan hacia las habilidades de pensamiento de orden superior (HOTS, por las siglas inglesas High Order Thinking Skills), de ahí las competencias clave para su futuro (Meyer, Coyle, Halbach, Schuck \& Ting, 2015; Coyle, 2015; Kovacikova \& Education, 2018). $Y$ estos son exactamente los objetivos previstos para el aprendizaje de contenido por AICLE (Marsh, 2002; Coyle, 2002; Marsh \& Frigols Martín, 2012) y por el nivel de su motivación, más alto que el que muestran los estudiantes no AICLE (Marsh, 2002; Lorenzo, Casal \& Moore, 2010; Marsh \& Frigols Martín, 2012; Banegas, 2012; Czura \& Papaja, 2013; Guillamón-Suesta et al, 2015; Meyer, Coyle, Halbach, Schuck \& Ting, 2015; Pérez Cañado, 2016; Cinganotto, 2016; Dallinger, Jonkmann, Hollm \& Fiege, 2016; Goris, Denessen \& Verhoeven, 2017; Kovacikova \& Education, 2018). Con todo, en muchos países o escuelas europeas existen criterios de selección para acceder al AICLE, y sus pruebas a menudo son sobre conocimientos previos y habilidades ya adquiridas en los temas y el idioma sobre los que se prevé articular la experiencia AICLE (Eurydice, 2017a), lo cual puede acabar desvirtuando los resultados de evaluación, sin duda.

En la práctica, AICLE se implementa mediante 'a plethora of models or variants which can be identified within it [...] dependent on a series of factors or parameters', como se indica por Pérez Cañado (2016, p.14). A veces, los modelos vienen sugeridos por las autoridades educativas (Eurydice, 2017a) sobre, por ejemplo, qué idioma extranjero o minoritario se va a utilizar y su presencia proporcional (Nikula et al., 2013). Pero estos amplios modelos son puestos en práctica por los docentes de materias no lingüísticas, solos o en equipo con compañeros de idiomas o otros (Marsh, 2002; Banegas, 2012; Marsh \& Frigols Martín, 2012; Nikula et al., 2013; Guillamón-Suesta et al., 2015; Agolli, 2015; Cinganotto, 2016; van Kampen, Meirink, Admiraal \& Berry, 2017). Estos determinan: 
- el contenido y la lengua vehicular, así como los objetivos didácticos (Marsh \& Frigols Martín, 2012; Agolli, 2015; Meyer et al, 2015; Coyle, 2015), con el fin de elegir los temas de su asignatura o de diferentes materias (Coyle, 2015);

- las estrategias didácticas (Banegas, 2012), en función de:

- el contexto de la clase (Dalton-Puffer et al, 2014; Agolli, 2015) y los objetivos grupales, subgrupales o individuales (Marsh, 2002; Marsh \& Frigols Martín, 2012);

- el proyecto educativo de centro (Llinares \& Morton, 2010; Doiz, Lasagabaster \& Sierra, 2014; Dalton-Puffer et al., 2014; Pérez Cañado, 2016; Kovacikova \& Education, 2018);

- su formación previa (Marsh, 2002; Bruton, 2011; Dalton-Puffer et al., 2014; Cinganotto, 2016; Kovacikova \& Education, 2018);

- su propio dominio lingüístico en L1 y L2 (Meyer, Coyle, Halbach, Schuck \& Ting, 2015; Pérez Cañado, 2016) y su relación con las habilidades de aprendizaje (Dalton-Puffer, 2007; Muñoz-Luna, 2014; Doiz et al., 2014);

- factores emocionales, como la motivación y el entusiasmo de estudiantes y profesores (Doiz, Lasagabaster \& Sierra, 2014; Dallinger, Jonkmann, Hollm \& Fiege, 2016).

Para poner en práctica AICLE, los profesores primero sopesan diversas opciones teóricas y estratégicas, que inciden en la práctica en los resultados de los estudiantes, en el rol del profesor dentro de la clase, en los recursos didácticos y también en la disposición de los estudiantes y estaciones de trabajo en la clase. Los modelos más difundidos son cuatro:

1. El Marco 4C visto antes (Coyle, 1999), que expone la integración de contenido-cognicióncomunicación-cultura para la planificación de los profesores en el AICLE. Está dirigido a hacer que los estudiantes aprendan de manera personalizada, pero en particular 'it was a means of enabling both language teachers and subject teachers to be supported in a basic understanding that CLIL was not about deciding which content or which language needed to be taught but involved a much deeper and complex conceptualisation of learning including cognitive demands and intercultural understanding' (Coyle, 2015). A este modelo, Agolli (2015) le añade el contexto. Y, finalmente, se completa con el tríptico del lenguaje (language triptyc) (Coyle, Hood, Marsh, 2010), con el fin de crear una unificación visual de diferentes funciones lingüísticas necesarias en una implementación AICLE: la lengua vehicular obligatoria para el contenido y compatible con él; en definitiva, un sistema lingüístico que enriquece el contenido (Coyle, 2015).

2. La Pirámide $A I C L E$ se basa en el marco $4 C$ y fue desarrollada como una herramienta de planificación integradora para los diseñadores de materiales y planificadores de implementaciones AICLE (Meyer, 2010). Propone puntos secuenciales a tener en cuenta para planificar estos módulos: la selección del tema, el núcleo de las sesiones; la elección de entradas multimodales a través de actividades transmedia, la elección de input-scaffolding y el conocimiento de las técnicas de estudio necesarias; el diseño de las tareas, con el fin de llevar a los estudiantes a alcanzar las HOTS y los resultados de comunicación e interacción a través de output-scaffolding; el ejercicio AICLE, es decir, la revisión de los contenidos clave y elementos del lenguaje (Meyer, 2010).

3. El modelo de alfabetización múltiple (Pluriliteracies model) nació como un proyecto del CELM (Centro Europeo de Lenguas Modernas) con el fin de 'sensitise teachers towards pluriliteracy as an end-goal in both content education and language learning and provide them with a guide 
towards more literacy-sensitive classroom practices' (Meyer et al., 2015). Su mapeo visual de progreso ha sido desarrollado por Austrian Graz Group, una colaboración de expertos internacionales, con el fin de trazar el progreso de los alumnos no solo en conocimiento del contenido y lenguaje integrado, sino también en las competencias específicas, lo que implica las interpretaciones y la construcción del conocimiento (Meyer et al., 2015) a través de los idiomas y la cultura, mostrando, por lo tanto, el desarrollo conceptual del contexto y el desarrollo del lenguaje (Coyle, 2015).

4. El modelo tridimensional (Ball, Kelly, Klegg, 2015), en el que la interacción de conceptoprocedimiento-lenguaje se planifica con facilidad a través del marco 4C, así como por medio de la comprensión de los términos y por su aplicación. De hecho, la cognición, por ejemplo, está aquí mezclada, como en las lecciones L1, tanto en el concepto, que es el contenido por enseñar, y el procedimiento, es decir, la estrategia como enseñamos (Ball, Kelly, Klegg, 2015). Por ello se propuso la idea de un 'nuevo profesor híbrido', que debe hacer hincapié en una u otra dimensión para diferentes objetivos, en diferentes situaciones o contextos, pero que antes debe también adquirir algunas competencias de los profesores de lenguas extranjeras, ya que el lenguaje es clave para un alto rendimiento del alumnado, a pesar de que la prioridad siempre debe ser el contenido (Ball, Kelly, Klegg, 2015).

Estos cuatro modelos tienen raíces constructivistas o socio-constructivistas (Dalton-Puffer, 2011; Marsh \& Frigols Martín, 2012; Nikula et al., 2013; Muñoz-Luna, 2014; Coyle, 2015; Meyer et al., 2015; Agolli, 2015; Hüttner \& Smit, 2017; van Kampen, Meirink, Admiraal \& Berry, 2017), que se traduce entre otros en una fuerte implicación de los estudiantes en todo el proceso de aprendizaje, y bajo una perspectiva inclusiva y, por concepto, ecléctica, que incluye diferentes métodos y concepciones interdisciplinares (Muñoz-Luna, 2014).

También cabe destacar que cualquier modelo puede ser implementado de diferentes maneras, como por ejemplo a través de un modelo monodisciplinario/multidisciplinario/interdisciplinario; $y$, por tanto, a veces también en las asignaturas de lenguas extranjeras (Banegas, 2012); en algún lugar con una fase pre-AICLE (Czura \& Papaja, 2013; Agolli, 2015); dirigida por el contenido o el lenguaje (Marsh, 2002; Banegas, 2012; Coyle, 2015; Pérez Cañado, 2016); conducido por el lenguaje, como un AICLE soft, o conducido por el contenido, como un AICLE hard (Ball, Kelly, Klegg, 2015); a través de un uso masivo de las TIC (Meyer, 2010; Cinganotto, 2016; Fokides \& Zampouli, 2017; Kovacikova \& Education, 2018), o también sin el uso tecnologías (por ejemplo, Czura \& Papaja, 2013).

Según la literatura, otras de las preocupaciones sobre la implementación AICLE podrían ser la 'inseguridad y la desaceleración del ritmo del curso' (Guillamón-Suesta et al, 2015; Ver también Dallinger, Jonkmann, Hollm \& Fiege, 2016), debido a la atención concurrente al contenido y al idioma por parte de los profesores procedentes de la educación general (Guillamón-Suesta et al, 2015.); la falta de materiales, a menudo creados de forma autónoma por los profesores AICLE (Meyer, 2010; Banegas, 2012; Dallinger, Jonkmann, Hollm \& Fiege, 2016; Cinganotto, 2016); o la adopción de diferentes estrategias dentro de las clases grupales a diferencia de la tradicional, centrada en el profesor (Nikula et al, 2013;. Czura \& Papaja, 2013; Muñoz-Luna, 2014).

Y, además, los profesores especializados en AICLE, que saben que tienen que evaluar los resultados complejos (Muñoz-Luna, 2014; Coyle, 2015) y su importancia para el crecimiento de los alumnos, observan que el sistema de evaluación tradicional no tiene en cuenta el proceso del aprendizaje y la adquisición de las habilidades metacognitivas (Meyer, 2010; Muñoz-Luna, 2014) en diversos contextos, como diversas materias no lingüísticas determinan (Marsh, 2002; Nikula et al, 2013; Muñoz-Luna, 2014), y no se consideran todos los resultados al mismo tiempo durante la ejecución. Estos resultados, de hecho, se encuentran en diferentes campos: 
- especificamente lingüísticos en varios niveles: como la producción oral y escrita, el conocimiento y el uso del lenguaje académico, la construcción de significados/conocimiento, etc. (Nikula et al, 2013; Muñoz-Luna, 2014);

- la autonomía (Muñoz-Luna, 2014), necesaria para investigar y profundizar también a través de una lengua extranjera (Marsh, 2002; Coyle, 2002; Dallinger et al, 2016) y a través de diferentes perspectivas (Marsh, 2002; Coyle, 2002; Muñoz-Luna, 2014);

- la gestión de contenidos y la creación de conocimientos por parte de los alumnos (Meyer, 2010; Muñoz-Luna, 2014); la interacción y la cooperación con los compañeros de clase o profesorado (Meyer, 2010; Nikula et al, 2013; van Kampen., Admiraal \& Berry, 2016; Hüttner \& Smit, 2017);

- la ciudadanía mundial (Goris, Denessen \& Verhoeven, 2017) y, en particular, europea e intercultural (Meyer, 2010; Lorenzo, Casal \& Moore, 2010; Van Kampen, Admiraal \& Berry, 2016);

- la competencia digital (Meyer, 2010; Marsh \& Frigols Martín, 2012; Ramírez-Verdugo, 2012; Cinganotto, 2016);

- la motivación (Doiz et al., 2014) para estudiar el tema implicado en AICLE, no solo para la lengua extranjera utilizada (Nikula et al., 2013).

Por lo tanto, una evaluación limitada solo al contenido o a las destrezas lingüísticas (como se suele hacer en los análisis nacionales) (Banegas, 2012), puede sugerir menores aprendizajes en el contenido específico curricular de las asignaturas, a pesar de que existan la mejora evidente de otras competencias (Meyer, Coyle, Halbach, Schuck \& Ting, 2015; Dallinger et al, 2016), el logro de competencias de alfabetización multiple (Coyle, 2015) o la alfabetización transmediática superior. Por otra parte, entre los principales retos del AICLE, la literatura consagra la creación de un completo sistema de evaluación global de contenido y lenguaje (Dalton-Puffer, 2011; Banegas, 2012), con todos los elementos anteriores y suficientemente flexible y ágil para adaptarlo a las necesidades individuales, grupales y contextuales (Marsh, 2002; Agolli, 2015).

\section{Discusión}

Desde su comparación hasta ahora, el AICLE ha sido fuertemente seguido por el área de lengua y lingüística, a pesar de que su implementación debe ser enseñada en su mayoría por profesores no especializados en lingüística (Nikula et al., 2013). Buena prueba de esto se puede encontrar en las Universidades de Europa, donde la investigación sobre AICLE se radica en las facultades de Lenguas Extranjeras, y donde también se ofrece la formación para docentes en AICLE. Esto se debe, como se ha visto, a las raíces de AICLE de IBC canadiense (Dalton-Puffer, 2011; Nikula et al, 2013), donde el inglés y el francés son los dos idiomas del país, y a las directivas de la Comisión Europea (por ejemplo, la Resolución de 2008), con el fin de promover la ciudadanía europea también a través del multilingüismo (European Commission, 1995; Council \& Council, 2002; Resolution, 2008).

Pero, por ejemplo, los profesores de historia pueden preguntarse: ¿por qué debería concernirme esto? Y ¿por qué debería adoptar una forma compleja de enseñanza, a través de otro idioma, sin reglas específicas que aplicar, trabajando más de lo habitual para construir mis propios recursos didácticos, poniendo en riesgo la evaluación positiva de los estudiantes en el examen regular al final de la escuela secundaria? Estas son las preguntas frecuentes que debemos responder, a fin de consolidar la implementación del AICLE, de tal modo que no solo enraíce entre los ya interesados (Marsh, 2002; 
Lorenzo, Casal \& Moore, 2010; Dalton-Puffer, 2011; Pérez Cañado, 2016). Y en la literatura hay un importante vacío en este sentido, desde el punto de vista de los profesores.

Se puede decir que, en este asunto, para un profesor, el punto de partida es enlazar con las directivas europeas, que expresan las necesidades prácticas y sobre todo económicas de los países de la Unión, así como su demanda de un proceso de modernización en la educación para garantizar la ciudadanía europea de los estudiantes. Es muy interesante ver cómo el European White Paper (European Commission, 1995), (no es reciente, pero sigue siendo válido para el análisis de necesidades), en una de sus funciones, llamar la atención al asunto crucial de la enseñanza para promover la innovación, resalta enérgicamente que una excesiva estandarización del conocimiento prevalece como una cuestión de dominio de un sistema de razonamiento deductivo basado en conceptos abstractos, lo que no es ahora propio para desarrollar habilidades directivas, tales como el juicio de las circunstancias o la toma de decisiones en un contexto internacional y tecnológico, derivado de la sociedad de información, que es precisamente nuestra sociedad ahora más que nunca. Por ello, la escuela no solo debe permitir la capacidad crítica para ser desarrollada en todos los niveles, entre los alumnos y los profesores, sino que también debe alentarla (Comisión Europea, 1995). Para alcanzar este punto, el AICLE puede ser la clave: de hecho, necesitamos un profesorado capaz de planificar paso a paso la forma de involucrar a los estudiantes en las HOTS, como se ve en todos los modelos de AICLE, como un ambiente de aprendizaje básicamente abierto a diferentes estrategias (Marsh \& Frigols Martín 2012; Coyle, 2015; Cinganotto, 2016; Goris et al, 2017), en función de la clase grupal, las diferentes tipos de aplicaciones elegidas y sobre todas las variables sobre la evaluación.

Por lo tanto, la falta de normas específicas para poner en práctica propuestas AICLE, que a menudo se traduce en flexibilidad (Coyle, Hood, Marsh, 2010), podría ser una ventaja, a fin de elegir la mejor manera de fomentar las habilidades o conocimientos de los estudiantes en cada momento o situación, adecuado a los distintos tipos de profesorado o su actitud con respecto a una estrategia particular por aplicar (van Kampen, Meirink, Admiraal \& Berry, 2017). Este es el enfoque correcto para estimular la motivación, que se ha demostrado tanto en los profesores como en los alumnos (Marsh \& Frigols Martín, 2012; Nikula et al., 2013; Banegas, 2012; Czura \& Papaja, 2013; Guillamón-Suesta et al., 2015; Meyer, Coyle, Halbach, Schuck \& Ting, 2015; Pérez Cañado, 2016; Cinganotto, 2016; Dallinger, Jonkmann, Hollm \& Fiege, 2016; Goris, Denessen \& Verhoeven, 2017; Kovacikova \& Education, 2018), más allá del mayor esfuerzo para adaptar las lecciones y los materiales una y otra vez para los profesores, así como hacer frente a los nuevos contenidos en un idioma extranjero para los estudiantes. Como decíamos, a pesar de los elementos disuasores, AICLE puede suponer una ventaja, ya que los profesores y estudiantes tienen que cooperar efectivamente para crear sus propios materiales y lecciones adaptadas (Banegas, 2012).

Obviamente, esto tiene que respetar el alma de AICLE, que nació bajo la idea de la integración (Marsh, 2002), no solo sobre el contenido y el lenguaje, sino para dar valor a las diferentes contribuciones de los estudiantes de diferentes niveles en una clase, para hacer que los estudiantes triunfen, no solo de forma individual, sino en cooperación, en su más alto nivel de HOTS, (Meyer, 2010;; Marsh \& Frigols Martín, 2012 Dallinger et al, 2016), elementos que aprovecharán toda su vida: es decir, crear la un modelo de educación propio de nuestros tiempos, centrado en el alumnado. De hecho, se puede observar que los cuatro marcos AICLE tienden a ser la base de un nuevo tipo de escolarización, en el que, por un lado, los profesores estimulan fuertemente el desarrollo de las facultades críticas, andamiando el proceso de crecimiento de los alumnos a lo largo del contexto de desarrollo conceptual y el desarrollo del lenguaje (Coyle, 2015); por otro lado, los estudiantes son los personajes principales, implicados en todo el proceso de aprendizaje (Muñoz-Luna, 2014), lo que lleva a la exposición oral y escrita para la creación de sentido, la construcción del conocimiento y la cooperación con los compañeros de clase (Dalton-Puffer, 2011). En este punto, no es relevante si todos los datos mostraran grandes diferencias o no entre las clases AICLE e inglés como lengua extranjera, así como las clases tradicionales para una asignatura proporcionan a los estudiantes un conocimiento más teórico y estandarizado, con el fin, en primer lugar, de simplificar la evaluación. La eficacia se mide, como la Comisión Europea viene pidiendo, en cuanto a 
la calidad del conocimiento, es decir, de la importancia del proceso a lo largo de cada estudiante 'aprender a aprender', a través de:

- una amplia adquisición de base de conocimientos y habilidades metodológicas, ya que "enable people to find their way in the information society, that is to say to be able to interpret critically the images and information they receive from a variety of sources" (European Commission, 1995, p.11). En otras palabras, ahora más que entonces, es urgente comenzar a partir del dominio de la lectura, la escritura y la aritmética, a veces apenas aprendidas, para llegar a lograr habilidades transmediáticas (Meyer, 2010), como en la actualidad se les llama. La investigación siempre en curso sobre AICLE, como se ha visto, se ha establecido de estas necesidades durante los últimos 20 años, lo que subraya la importancia de la adquisición de contenidos, junto con el proceso cognitivo, y ha proporcionado modelos para mejorar todo esto. Los cuatro modelos AICLE más extendidos se han estado estableciendo de esta manera. Además, el sentimiento de ser parte de la sociedad de la información para los alumnos requiere ahora el uso de las TIC durante el proceso de aprendizaje (Marsh \& Frigols Martín, 2012; Ramírez-Verdugo, 2012; Cinganotto, 2016), como suele ocurrir en la implementación de AICLE, y todos buscan recursos sobre diversos temas y crean 'productos' personales de conocimiento, es decir, tareas personales o grupales (Meyer, 2010; Dalton-Puffer, 2011), a través de las muchas herramientas disponibles en línea que un profesor o un estudiante pueden elegir (Ramírez -Verdugo, 2012).

- el fortalecimiento de las destrezas sociales, como la cooperación, la creatividad y la búsqueda de la calidad (Comisión Europea, 1995), con el fin de ser capaces de encajar en el mundo del trabajo. Como se ve, AICLE se implementa para lograr importantes habilidades de alto nivel para la vida del alumnado y su vida laboral futura (Gaisch et al, 2017), básicamente, adoptando enfoques socio-constructivistas y basados en tareas (Muñoz-Luna, 2014; Meyer, 2010), para aprovechar el potencial de interacción de los estudiantes (Llinares \& Morton, 2010; Hüttner \& Smit, 2017), para hacerlos conseguir productos creativos concretos y comunes a cada grupo y para cada tema/módulo, para evaluar su comprensión profunda (Dalton-Puffer, 2011; Agolli, 2013; Coyle, 2015;. Dallinger et al, 2016), la propia gestión del conocimiento que han adquirido durante su interacción para una tarea común (Nikula et al., 2013; Meyer et al., 2015), y de ahí la calidad.

- las habilidades lingüísticas, en primer lugar, en la lengua materna, que es la base del aprendizaje y la interacción y que estimula, en un enriquecimiento mutuo, el aprendizaje de las lenguas extranjeras (Lorenzo, Casal \& Moore, 2010; Dalton-Puffer, 2011; Guillamón-Suesta et al, 2015; Coyle, 2015; Agolli, 2015). Por otra parte, está demostrado que el aprendizaje de los idiomas extranjeros se ha convertido en una condición previa para que los ciudadanos de la Unión Europea puedan beneficiarse de las oportunidades profesionales y personales que se les ofrecen en el mercado único sin fronteras; es, de hecho, la clave para saber de otras personas; ayuda a construir el sentimiento de ser europeo con toda su riqueza y diversidad cultural y de entendimiento entre los ciudadanos de Europa; abre la mente, estimula la agilidad intelectual y, por supuesto, expande el horizonte cultural de las personas (European Commission, 1995). Por ello, los profesores de asignaturas no lingüísticas, y nosotros entre ellos, tenemos que admitir los amplios y validados beneficios derivados del estudio de las lenguas extranjeras (Resolution 2008) y la atención importante que debe darse a la dimensión lingüística de un tema específico para llegar también a otros objetivos, en particular, los cognitivos (por ejemplo, Lorenzo, Casal \& Moore, 2010; Nikula et al, 2013; Meyer, Coyle, Halbach, Schuck \& Ting, 2015). De hecho, a menudo la formación de profesores para AICLE es prerrogativa de los departamentos de Inglés de las Universidades, como lengua extranjera AICLE más importante. El AICLE, en este campo, es la razón por la que se implementa en Europa (Resolution, 2008; Eurydice, 2017a), y permite a los estudiantes aprender lenguas extranjeras en diversos contextos que corresponden a diferentes asignaturas (Marsh, 2002; Marsh \& Frigols Martín, 2012 ; Banegas, 2012; Ramírez- 
Verdugo, 2012; Agolli, 2013), pero con diferentes lenguajes académicos (Llinares \& Morton, 2010; Dalton-Puffer, 2011; Meyer et al., 2015; Kovacikova \& Education, 2018), lo que les permite investigar de manera autónoma en al menos un idioma extranjero y en diversas bibliografías y fuentes (Doiz et al, 2014;. Pfenninger, 2014; Guillamón-Suesta et al, 2015; Gaisch et al, 2017). Por otra parte, el AICLE, como se ha visto, debe tener en cuenta el elemento cultural (Coyle, 1999), el cual es expandir los horizontes de los estudiantes, dejarlos crecer en una perspectiva multicultural o, mejor, intercultural (Ramírez-Verdugo, 2012) con el descubrimiento de las raíces comunes europeas. Es importante también citar el hecho de que el programa europeo Erasmus+, que ha apoyado el AICLE como una estrategia importante para el aprendizaje de idiomas (Cinganotto, 2016; Eurydice, 2017a; Kovacikova \& Education, 2018), ha venido financiando las movilidades de los estudiantes, lo que resulta realmente importante para hacer que se sientan ciudadanos europeos, para conocer a personas extranjeras con su diversidad y para darles una idea de las oportunidades personales en el extranjero.

Por último, puede considerarse que el AICLE enriquece tanto la lengua materna como el idioma de destino (Marsh 2002), así como la motivación para aprender (Doiz et al., 2014), y permite que los estudiantes obtengan mejores resultados si tienen que construir significados con el lenguaje académico (Meyer et al, 2015). Y, por su parte, las explicaciones (Llinares \& Whittaker, 2007; Llinares \& Morton, 2010), pasan de un idioma a otro, desde el contexto de un tema a otro. De hecho, esto podría ser mejor estratégicamente para poner en práctica AICLE en más de un tema curricular, posiblemente en forma interdisciplinaria, con el fin de adoptar diferentes entornos de aprendizaje, mientras aún no consideramos la inmersión total en el idioma extranjero (en la que el inglés es la mejor opción para sus crecientes habilidades escritas y orales), de tal modo que no se elimine el constante esfuerzo cognitivo constante de la construcción del conocimiento en ambos idiomas y en diferentes contextos significativos. De esta manera, un estudiante, desempeñándose mejor en un tema, puede cooperar con los que serían mejor en otro, y ambos mejoran su conocimiento del contenido (Muñoz-Luna, 2014). El objetivo de la fluidez en el idioma extranjero, a menudo declarado en AICLE (Marsh, 2002; Dalton-Puffer, 2011), aunque más fácil de lograr en los programas de inmersión (Pfenninger, 2014), podría también mejorar en modelos interdisciplinarios, donde exposiciones más largas a la lengua extranjera involucran a los estudiantes y el equipo de enseñanza a pasar más tiempo durante el año académico que en un solo tema, aparte de dar la visión cruzada curricular de cada tópico y la seguridad necesaria de los conocimientos para comunicarse (Llinares \& Morton, 2010; Dalton-Puffer, 2011).

\section{Conclusión}

Esta revisión se ha tratado de ofrecer una definición atractiva del AICLE para el profesorado de materias no lingüísticas; $y$, a partir de las evidencias empíricas, analizar cuán atractivo lo hacen no solo para profesores de lenguas extranjeras. De acuerdo con Dalton-Puffer (2011, p. 196), "CLIL provides a space for language learners that is not geared specifically and exclusively to foreign language learning but at the same time is predefined and prestructured in significant ways by being instructional and taking place within the L1 matrix culture". Y en este propósito, AICLE no es selectivo, como se ha dicho en ocasiones (Bruton, 2011), porque los estudiantes están involucrados y motivados para seguir adelante con sus procesos personales. Se puede decir que el AICLE se define como un entorno educativo abierto y significativo, lejos de las políticas descendentes o imposiciones metodológicas (Agolli, 2013), y podría suponer un revulsivo para el profesorado, especialmente en equipo, para cambiar los modelos didácticos tradicionales y la evaluación de los estudiantes, que hoy en día necesitan diferentes habilidades para su vida a diferencia del pasado $y$, por tanto, diferentes enfoques y herramientas para lograr sus objetivos. Si este es el objetivo, los resultados de la aplicación del AICLE pueden considerarse al menos interesantes para cualquier profesor, a pesar de su necesidad de formación. De hecho, su complejidad durate la 
planificación, la preparación de las clases, la elección de las herramientas, el uso de un idioma extranjero, el proceso de evaluación son los desafíos que permite la construcción de una escuela abierta al mundo exterior, centrada en el estudiante y por tanto más eficaz. Y, al tiempo, también demanda profesores híbridos (Ball, Kelly, Klegg, 2015), que sean capaces de andamiar a los alumnos en su camino hacia el conocimiento y aprovechar el amplio potencial del aprendizaje de idiomas en contexto. Los profesores AICLE, de esta manera y especialmente en tándem con los profesores de idiomas, podrán conseguir que el alumnado sea capaz de obtener su ciudadanía europea, a través de sus habilidades de pensamiento, lingüísticas y sociales adquiridas a través del entorno abierto de AICLE.

Sin duda, se trata de un objetivo futuro exigente, cuya consecución requiere considerar la evaluación como el núcleo de investigaciones posteriores, que también debe incluir al menos la formación del profesorado y las diferentes estrategias en función de las diversas áreas disciplinares.

\section{Referencias bibliográficas}

Agolli, R. (2013). A Penetrating Content and Language Integrated Learning (CLIL) Praxis in Italian Mainstream Education: Stemming Novelites and Visions. Research Papers in Language Teaching and Learning, 4(1), 138-157.

Agolli, R. (2015). Content and language symbiosis in a maieutic, translanguaging pattern (CLSL): An exploratory practice in Italy. Latin American Journal of Content and Language Integrated Learning, 8(2), 161-183. https://doi.org/10.5294/laclil.2015.8.1.5

Ball, P., Kelly, K., \& Clegg, J. (2015). Putting CLIL into Practice. Oxford: Oxford University Press.

Banegas, D. L. (2012). Integrating content and language in English language teaching in secondary education: Models, benefits, and challenges. Studies in Second Language Learning and Teaching, 2(1), 111-136. https://doi.org/http://dx.doi.org/10.14746/ssllt.2012.2.1.6

Barcelona European Council. (2002). Presidency Conclusions. Barcelona European Council, 35, 0-72. Retrieved from http://ec.europa.eu/invest-inresearch/pdf/download_en/barcelona_european_council.pdf

Biçaku, R. Ç. (2011). CLIL and teacher training. Procedia-Social and Behavioral Sciences, 15, 3821-3825. https://doi.org/10.1016/j.sbspro.2011.04.379

Bruton, A. (2011). Is CLIL so beneficial, or just selective? Re-evaluating some of the research. System, 39(4), 523-532. https://doi.org/10.1016/j.system.2011.08.002

Cinganotto, L. (2016). CLIL in Italy: a general overview. Latin American Journal of Content \& Language Integrated Learning, 9(2), 374-400. https://doi.org/10.5294/laclil.2016.9.2.6

Council of the European Union. (2002). Resolution of 14 February 2002 on the promotion of linguistic diversity and language learning in the framework of the implementation of the objectives of the European Year of Languages 2001, 50(45), 1-3. http://europa.eu/eurlex/pri/en/oj/dat/2002/c_050/c_05020020223en00010002.pdf 
Coyle, D. (1999). Theory and planning for effective classrooms: supporting students in content and language integrated contexts. Learning through a Foreign Language, 46-62.

Coyle, D. (2002). Relevance of CLIL to the European Commission's language learning objectives. CLIL/EMILE the European Dimension. In Marsh, D. (2002). The European Dimension: Actions, Trends and Foresight Potential, 27-28. Strasbourg: European Commission. UniCOM, University of Jyvaskyla. Retrieved from https://jyx.jyu.fi/handle/123456789/47616

Coyle, D., Hood, P., \& Marsh, D. (2010). CLIL: Content and language integrated learning. Cambridge, England: Cambridge University Press.

Coyle, D. (2015). Strengthening integrated learning: Towards a new era for pluriliteracies and intercultural learning. Latin American Journal of Content and Language Integrated Learning, 8(2), 84-103. https://doi.org/10.5294/laclil.2015.8.2.2

Czura, A., \& Papaja, K. (2013). Curricular models of CLIL education in Poland. International Journal of Bilingual Education and Bilingualism, 16(3), 321-333. https://doi.org/10.1080/13670050.2013.777388

Dallinger, S., Jonkmann, K., Hollm, J., \& Fiege, C. (2016). The effect of content and language integrated learning on students' English and history competences - Killing two birds with one stone? Learning and Instruction, 41, 23-31. https://doi.org/10.1016/j.learninstruc.2015.09.003

Dalton-Puffer, C. (2011). Content-and-language integrated learning: From practice to principles? Annual Review of Applied Linguistics, 31, 182-204. https://doi.org/10.1017/S0267190511000092

Dalton-Puffer, C., Llinares, A., Lorenzo, F., \& Nikula, T. (2014). "You can stand under my umbrella": Immersion, CLIL and bilingual education. A response to Cenoz, Genesee \& Gorter (2013). Applied Linguistics, 35(2), 213-218. https://doi.org/10.1093/applin/amu010

Doiz, A., Lasagabaster, D., \& Sierra, J. M. (2014). CLIL and motivation: The effect of individual and contextual variables. Language Learning Journal, 42(2), 209-224. https://doi.org/10.1080/09571736.2014.889508

European Commission. (1995). White paper on education and learning - Teaching and learning: towards $\begin{array}{lllll}\text { the learning } & \text { society, } & \text { Retrieved }\end{array}$ http://europa.eu/documents/comm/white_papers/pdf/com95_590_en.pdf

European Council. (2008). Council resolution of 21 November 2008 on a European strategy for multilingualism. 2008/C 3210/01. C320. http://eur-lex.europa.eu/legalcontent/DE/TXT/?uri=celex:32008G1216(01)

Eurydice. (2017a). Key Data on Teaching Languages at School in Europe. 2017 Edition. Luxembourg: EU Publications. https://doi.org/10.2797/839825

Eurydice. (2017b). Key Data on Teaching Languages at School in Europe. 2017 Edition [brief edition]. Luxembourg: EU Publications. https://doi.org/10.2797/828497

Fokides, E., \& Zampouli, C. (2017). Content and Language Integrated Learning in Opensimulator Project. Results of a Pilot Implementation in Greece. Education and Information Technologies, 22(4), 1479-1496. https://doi.org/10.1007/s10639-016-9503-z

Gaisch, M., Rammer, V., Hruskova, L., Krátká, J., \& Mádlová, G. (2017). Content language integrated learning as a driver for enhanced graduate employability. A cross-cultural study between Austria and 
the Czech Republic. In M. Uberwimmer, M. Gaisch, R. Fureder \& Y. Costa (Eds.) 233, Cross-Cultural Business Conference 2017. Germany: Shaker Verlag GmbH. Retrieved from https://www.researchgate.net/publication/317013155_Content_Language_Integrated_Learning_as_a_Dr iver_for_Enhanced_Graduate_Employability_A_cross-

cultural_study_between_Austria_and_the_Czech_Republic

Goris, J., Denessen, E., \& Verhoeven, L. (2017). The contribution of CLIL to learners' international orientation and EFL confidence. Language Learning Journal, 1736, 1-11. https://doi.org/10.1080/09571736.2016.1275034

Guillamón-Suesta, F., Luisa, M., \& Renau, R. (2015). A critical vision of the CLIL approach in secondary education: A study in the Valencian Community in Spain. Latin American Journal of Content and Language Integrated Learning, 8(1), 1-12. https://doi.org/10.5294/laclil.2014.8.1.1

Kiely, R. (2011). Understanding CLIL as an innovation. Studies in Second Language Learning and Teaching, 1(1), 153-171. https://doi.org/10.14746/ssllt.2011.1.1.9

Kovacikova, E., \& Luprichova, J. (2018). A good CLIL practice among European educational institutions International, International Journal of Learning and Teaching. 10(1), 50-60. https://doi.org/10.18844/ijlt.v10i1.3145

Llinares, A., \& Morton, T. (2010). Historical explanations as situated practice in content and language integrated learning. Classroom Discourse, 1(1), 46-65. https://doi.org/10.1080/19463011003750681

Marsh, D. (1994). Bilingual Education \& Content and Language Integrated Learning. Paris: International Association for Cross-cultural Communication, Language Teaching in the Member States of the European Union (Lingua), University of Sorbonne.

Marsh, D. (2002). The European Dimension: Actions, Trends and Foresight Potential. Strasbourg: European Commission. UniCOM, University of Jyvaskyla. Retrieved from https://jyx.jyu.fi/handle/123456789/47616

Marsh, D., \& Frigols Martín, M. J. (2012). Introduction: Content and Language Integrated Learning. In C. A. Chapelle, The Encyclopedia of Applied Linguistics, Chichester, West Sussex, UK. https://doi.org/10.1002/9781405198431.wbeal0190

Meyer, O. (2010). Towards quality-CLIL: successful planning and teaching strategies. Pulso, 33(1), 11-29. Retrieved from http://revistapulso.cardenalcisneros.es/documentos/articulos/114.pdf

Meyer, O., Coyle, D., Halbach, A., Schuck, K., \& Ting, T. (2015). A pluriliteracies approach to content and language integrated learning - mapping learner progressions in knowledge construction and meaningmaking. Language, Culture and Curriculum. https://doi.org/10.1080/07908318.2014.1000924

Muñoz-Luna, R. (2014). From Drills to CLIL: The Paradigmatic and Methodological Evolution Towards the Integration of Content and Foreign Language. Profile, 16(1), 1657-790. https://doi.org/10.15446/profile.v16n1.37843

Nikula, T., Dalton-Puffer, C., \& García, A. L. (2013). CLIL classroom discourse: Research from Europe. Journal of Immersion and Content-Based Language Education, 1(1), 70-100. https://doi.org/10.1075/jicb.1.1.04nik

Pérez Cañado, M. L. (2016). From the CLIL craze to the CLIL conundrum: Addressing the current CLIL controversy. Bellaterra Journal of Teaching \& Learning Language \& Literature, 9(1), 9-31. https://doi.org/http://dx.doi.org/10.5565/rev/jtl3.667 
Pfenninger, S. E. (2014). The misunderstood variable: Age effects as a function of type of instruction. Studies in Second Language Learning and Teaching, 3(3), 529-556. https://doi.org/10.14746/ssllt.2014.4.3.8

Ramírez-Verdugo, M. D. (2012). From Research to Development on Virtual Language, Content and Intercultural Learning Across European Schools, In L. Bradley \& S. Thouësny (Eds.), CALL: Using, Learning, Knowing, EUROCALL Conference, Gothenburg, Sweden, 22-25 August 2012, 245-249. https://files.eric.ed.gov/fulltext/ED574909.pdf

Van Kampen, E., Meirink, J., Admiraal, W., \& Berry, A. (2017). Do we all share the same goals for content and language integrated learning (CLIL)? Specialist and practitioner perceptions of "ideal" CLIL pedagogies in the Netherlands. International Journal of Bilingual Education and Bilingualism, 1-17. https://doi.org/10.1080/13670050.2017.1411332 